\title{
Paper-based Biosensor and Its Applications
}

\author{
Prinjaporn Teengam ${ }^{1}$, Sudkate Chaiyo ${ }^{1}$, Suchanat Boonkaew ${ }^{1}$, Weena Siangproh $^{2}$, Charles S. Henry ${ }^{3}$, \\ Kurt Kalcher ${ }^{4}$, Orawon Chailapaku1 ${ }^{1,5, *}$ \\ ${ }^{1}$ Electrochemistry and Optical Spectroscopy Center of Excellence, Department of Chemistry, \\ Chulalongkorn University, Pathumwan, Bangkok, 10330, Thailand \\ ${ }^{2}$ Department of Chemistry, Faculty of Science, Srinakharinwirot University, Bangkok, 10110, Thailand \\ ${ }^{3}$ Department of Chemistry, Colorado State University, Fort Collins, Colorado 80523, United State \\ ${ }^{4}$ Institute of Chemistry, Analytical Chemistry, Karl-Franzens University, Universitätsplatz 1, Graz A- \\ 8010, Austria \\ ${ }^{5}$ National Center of Excellence for Petroleum, Petrochemicals, and Advanced Materials, \\ Chulalongkorn University, Pathumwan, Bangkok, 10330, Thailand \\ corawon@chula.ac.th
}

\begin{abstract}
:
Paper-based analytical devices (PADs) have recently received renewed interest for point-ofcare testing since they are simple, inexpensive, portable and disposable. They have been extensively used for applications ranging from environmental analysis to clinical diagnosis. Herein, we concentrated on the development of PADs for biomedical applications. First, paper-based biosensor coupled with electrochemical detection for non-enzymatic determination of glucose was developed. A screen-printed carbon electrode modified with cobalt phthalocyanine, graphene and an ionic liquid (CoPc/G/IL/SPCE) shows excellent conductivity and fast electron transfer kinetics. Additionally, this sensor was successfully applied to quantify glucose level in human serum.

Moreover, a label-free electrochemical DNA sensor based on pyrrolidinyl peptide nucleic acid (acpcPNA)-immobilized PAD was developed. acpcPNA was covalently immobilized onto partially oxidized cellulose paper and served as a sensor probe for Mycobacterium tuberculosis (MTB) detection. The paper-based electrochemical DNA sensor was used to quantify a synthetic oligonucleotide of MTB by measuring the electrochemical signal, corresponding to the fractional change in the charge transfer resistance $\left(R_{\mathrm{ct}}\right)$ obtained from electrochemical impedance spectroscopy (EIS). The acpcPNA probe exhibited very high selectivity for the complementary oligonucleotides over the single-base-mismatch, two-base-mismatch and non-complementary DNA targets. In Addition, this DNA sensor platform was successfully applied to detect the PCR-amplified of extracted MTB DNA from clinical samples. The concept of label-free electrochemical immunosensor was also used for Creactive protein (CRP) detection. SPCE was modified by electrodeposition of gold nanoparticle onto electrode surface following by the immobilization of anibody. The change of $R_{\mathrm{ct}}$ after the addition of CRP at the electrode surface was investigated by EIS. This proposed immunosensor provided a high sensitive platform without any interference such as bilirubin, bovine serum albumin, and myoglobin. Furthermore, this immunosensor was successfully applied in a human serum

Not only electrochemical detection, but we also developed paper-based biosensor combined with colorimetric assay for DNA detection. The colorimetric paper-based DNA sensor was used to determine Middle East respiratory syndrome coronavirus (MERS-CoV), MTB and human papillomavirus (HPV). AgNPs were used as a colorimetric reagent for DNA detection based on acpcPNA-induced nanoparticle aggregation. The DNA target can be used to detect the color change of AgNPs. In addition, the multiplex paper-based DNA sensor was designed for simultaneous detection of MERS-CoV, MTB and HPV. From these results, the proposed paper-based biosensors show the potential to be an alternative device for sensitive, low-cost, simple and disposable in the field of biomedical applications.
\end{abstract}

Key words: Paper-based biosensor, Glucose, Middle East respiratory syndrome coronavirus, Mycobacterium tuberculosis, Human papillomavirus, C-Reactive protein 